\title{
Molecular Cytogenetic Diagnostics of Marker Chromosomes: Analysis in Four Prenatal Cases and Long-Term Clinical Evaluation of Carriers
}

\author{
Pavel Tesner $^{\mathrm{a}}$ Marketa Vlckova $^{\mathrm{a}}$ Jana Drabova $^{\mathrm{a}}$ Jan Vseticka ${ }^{\mathrm{c}}$ \\ Anna Klimovad Jana Lastuvkovad Jana Zidovskab

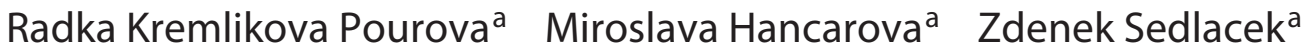 \\ Eduard Kocarek ${ }^{a}$ \\ aDepartment of Biology and Medical Genetics, Charles University 2nd Faculty of Medicine and University

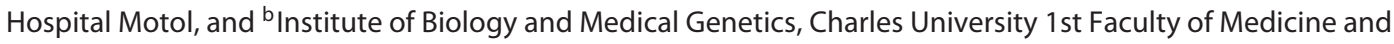 \\ General University Hospital, Prague, ${ }^{c}$ Genetika Ostrava s.r.o., Ostrava, and ${ }^{\mathrm{d}}$ Department of Medical Genetics, \\ Krajska Zdravotni, a.s. - Masaryk Hospital, Usti nad Labem, Czech Republic
}

\section{Established Facts}

- sSMCs are structurally abnormal chromosomes with a broad spectrum of clinical consequences from a normal phenotype to severe disorders.

- Their genotype-phenotype correlation is complicated and sometimes impossible, even postnatally.

- Prenatal identification of SSMCs is one of the most challenging situations in genetic counseling.

\section{Novel Insights}

- Our work contributes to the knowledge of management of prenatally detected sSMCs.

- It confirms FISH as a reliable method for fast sSMCs evaluation, particularly in mosaic and heterochromatic sSMCs.

- The prompt analysis of the sSMCs and the assessment of the prognosis can prevent unnecessary delays and uncertainty, allows informed decision making, and reduces unnecessary pregnancy terminations.

\section{Keywords}

$\mathrm{FISH} \cdot$ Genetic prognosis · Marker chromosome · Mosaicism · Prenatal diagnosis · Reproductive decision-making $\cdot$ Small supernumerary marker chromosome

\begin{abstract}
The prenatal finding of a small supernumerary marker chromosome (sSMC) is a challenge for genetic counseling. Our analytic algorithm is based on SSMC frequencies and multicolor FISH to accelerate the procedure. The chromosomal origin, size, and degree of mosaicism of the sSMC then deter-
\end{abstract}

Pavel Tesner

Department of Biology and Medical Genetics Charles University 2nd Faculty of Medicine and University Hospital Motol

V Úvalu 84, 15006 Prague 5 - Motol (Czech Republic)

E-Mail pavel.tesner@lfmotol.cuni.cz 
mine the prognosis. We illustrate the effectiveness on 4 prenatally identified de novo mosaic sSMCs derived from chromosomes $13 / 21, X, 3$, and 17 . Three sSMC carriers had a good prognosis and apparently healthy children were born, showing no abnormality till the last examination at the age of 4 years. One case had a poor prognosis, and the parents decided to terminate the pregnancy. Our work contributes to the laboratory and clinical management of prenatally detected SSMCs. FISH is a reliable method for fast SSMC evaluation and prognosis assessment; it prevents unnecessary delays and uncertainty, allows informed decision making, and reduces unnecessary pregnancy terminations.

(c) 2018 S. Karger AG, Basel

Small supernumerary marker chromosomes (sSMCs) are structurally abnormal chromosomes that cannot be unambiguously characterized using conventional chromosome banding [ISCN, 2016]. Their incidence is 1:1,340 in prenatal examinations and 1:2,250 in newborns [Liehr and Weise, 2007]. The difference between the 2 figures is partly caused by a higher spontaneous loss of fetuses with sSMCs [Kumar et al., 1997], substantially increased by selective termination of such pregnancies [Liehr and Weise, 2007]. Also, prenatally karyotyped cases cannot be considered unselected. Karyotyping leading to the prenatal identification of sSMCs is performed for various reasons, such as advanced maternal age (70.2\%), abnormal ultrasound findings (15.6\%), altered biochemical screening (6.3\%), and other reasons (7.9\%) [Liehr, 2017].

Most human sSMCs originate from acrocentric chromosomes. sSMCs from chromosome 15 [sSMC(15)] represent $22.9 \%$ of all cases, and those derived from other acrocentric chromosomes have a frequency of $23.8 \%$. sSMCs derived from nonacrocentric chromosomes account for $35.9 \%$ of cases, with isochromosomes $12 p$ and $18 \mathrm{p}$ being most frequently reported (because of their involvement in the Pallister-Killian syndrome and tetrasomy $18 \mathrm{p}$, respectively). sSMCs derived from chromosomes 8 and 9 are also relatively more frequent. A substantial group (11.2\%) comprises sSMCs which are supernumerary to the $45, \mathrm{X}$ Turner karyotype $\left(\mathrm{sSMC}^{\mathrm{T}}\right)$. $\mathrm{sSMC}^{\mathrm{T}} \mathrm{s}$ are mostly found in mosaics and almost all of them are derived from chromosomes X or Y. Some sSMCs can be neocentromeric (2.4\%), or multiple sSMCs can coexist in one cell (3.6\%) [Liehr, 2017]. The latter type is a unique subgroup with a much higher representation of nonacrocentrics, no inherited cases, much higher proportion of mosaic cases, and worse prognosis for the carriers [Hochstenbach et al., 2017].
The overall risk for an abnormal phenotype in de novo sSMC carriers is $13 \%$ [Warburton, 1991]. sSMCs derived from nonacrocentric autosomes have a higher risk (28\%), while the risk in sSMCs derived from acrocentric chromosomes other than chromosome 15 is lower (7\%) [Crolla et al., 1998]. Liehr and Weise [2007] reported about $70 \%$ carriers of de novo sSMCs as clinically normal. In cases with multiple sSMCs the proportion with a favorable prognosis was opposite (28.6\%), but a near-normal phenotype was reported even in cases with up to 7 sSMCs [Hochstenbach et al., 2017]. sSMCs(15) consisting only of pericentric heterochromatin and a part of the short arm are often familial and have a favorable prognosis. In contrast, sSMCs $(15)$ containing euchromatin including the Prader-Willi/Angelman critical region (PWACR) have a poor prognosis [Kleefstra et al., 2010]. $\mathrm{SSMC}^{\mathrm{T}} \mathrm{s}$ are associated with the phenotype of Turner syndrome or gonadal dysgenesis, but also with a normal phenotype.

sSMCs are usually identified using G-banding, which is still the basic step in prenatal diagnosis. Karyotyping also reveals the mosaic cases, and molecular cytogenetic techniques must then be applied to identify the origin of the sSMC. FISH is very important despite the recent boom of array techniques. Array comparative genome hybridization $(\mathrm{aCGH})$ can also determine the origin and size of the sSMCs [Gruchy et al., 2008; Marle et al., 2014]. However, it may fail in sSMCs formed exclusively of heterochromatin and in mosaic cases, and as a stand-alone test it can identify only about $75 \%$ of prenatal sSMC cases. These unidentified sSMCs are of a better prognosis because of the lack of euchromatin and because of a low level of mosaicism [Marle et al., 2014]. Nevertheless, such sSMCs could still alter meiosis and lead to fertility problems.

Here, we describe 4 cases of prenatally identified sSMCs of different origin and demonstrate the most suitable diagnostic approach in the individual situations which allowed us to promptly characterize the sSMC and to estimate the prognosis of the fetus, thus preventing unnecessary delays and uncertainty. The prognosis was markedly different in these 4 cases: case 1 carried a tiny sSMC derived from an acrocentric chromosome which are known to have a good prognosis; case 2 carried an SSMC derived from the $\mathrm{X}$ chromosome on the background of a mosaic $45, \mathrm{X} / 46, \mathrm{XX}$ karyotype; and cases 3 and 4 carried sSMCs derived from nonacrocentric chromosomes (3 and 17) which generally have a much more uncertain and worse prognosis. The parents of case 4 decided to terminate the pregnancy. In the 3 remaining cases we report the genotype-phenotype correlation obtained from our long-term follow-up of the carrier children. 
Fig. 1. FISH results of sSMCs (indicated by purple arrows). DNA counterstained using DAPI is shown in grey color. A Case 1, FISH for acrocentric chromosomes. Green signals show the alpha-satellite probe for chromosomes 13/21 and red signals show the alpha-satellite probe $14 / 22$. The sSMC is likely a bisatellited der(13/21). B Case 2, FISH for X chromosome. Green signals show the alpha-satellite probe $X$ and red signals show the XIST region probe (Xq13). C Case 3, cen-mFISH. Cut-outs show the sSMC(3) and a normal chromosome 5. From left to right: green FITC (on the sSMC), red Spectrum Orange (on chromosome 5), purple Texas Red (absent on both), yellow Cy5 (on the sSMC), and blue DEAC (on the sSMC). The combination of positive FITC, Cy5, and DEAC signals indicates the origin of the sSMC from chromosome 3 (labeling according to cen-mFISH scheme by Nietzel et al. [2001]). D Case 4, cen-mFISH (using the same labeling scheme as in C). Cut-outs show the sSMC(17) and a normal chromosome 5. From left to right: green FITC (on the sSMC), red Spectrum Orange (on chromosome 5), purple Texas Red (on the sSMC), yellow Cy5 (on the sSMC), and blue DEAC (absent on both). The combination of positive FITC, TxR, and Cy5 signals indicates the origin of the sSMC from chromosome 17.
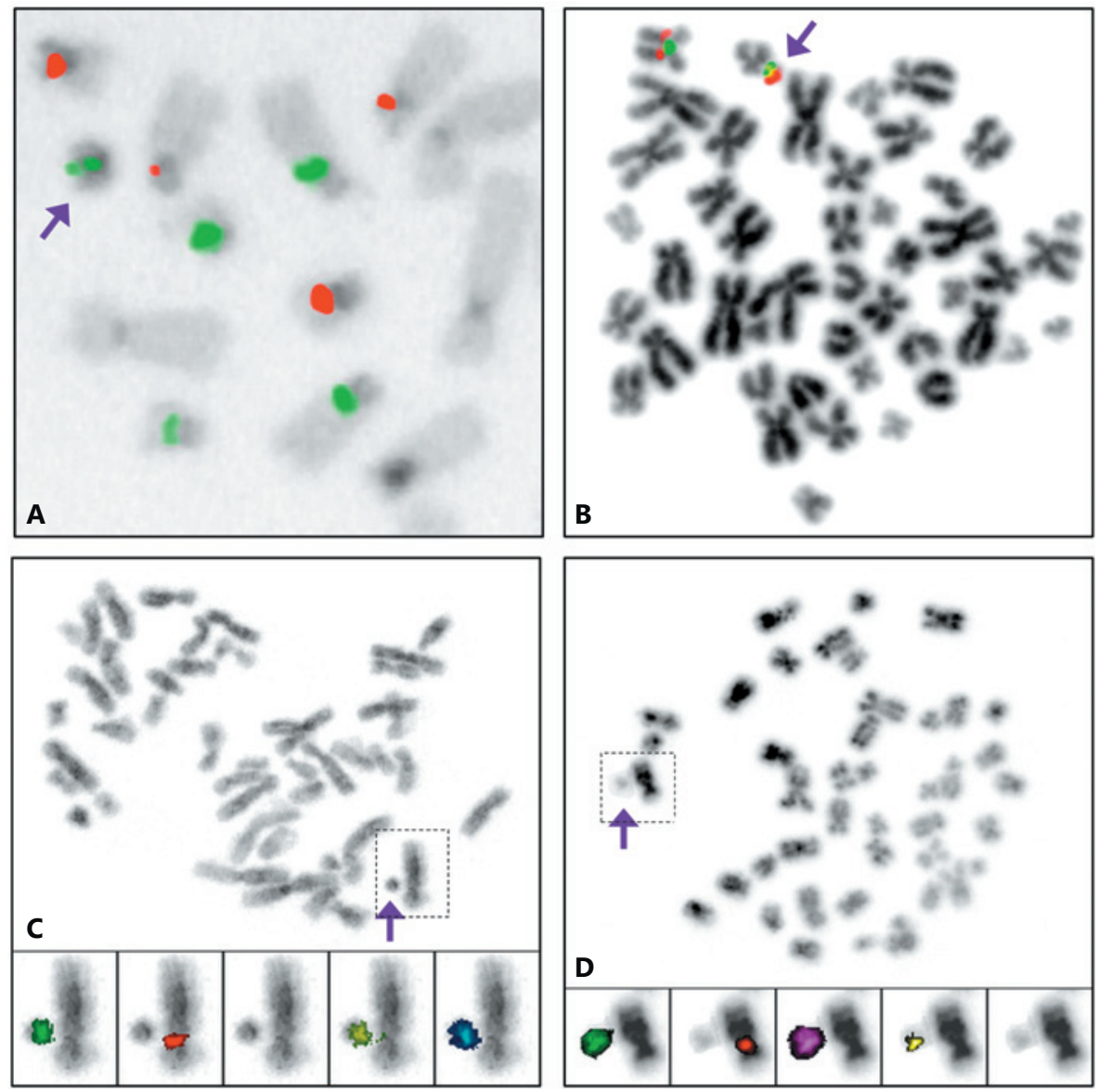

\section{Case Reports}

Case 1

Prenatal karyotyping revealed a male karyotype with a de novo sSMCin abouthalf of the cells (mos47,XY,+mardn[19]/46,XY[21]). The sSMC showed a signal of the chromosome 13/21 centromeric probe (Fig. 1A). FISH with painting probes for these chromosomes was negative, indicating the absence of a significant portion of euchromatin. aCGH was not performed and the exact origin of the sSMC is unclear.

Due to the favorable prognosis for carriers of sSMCs derived from acrocentric chromosomes, the parents decided to continue the pregnancy. A healthy boy was born in term with Apgar score (AS) of 10-10-10. The birth parameters were normal (shown for all patients together with postnatal growth parameters in online suppl. Table 1; see www.karger.com/doi/10.1159/000488790 for all online suppl. material). Postnatal karyotyping confirmed the prenatal finding ( $\operatorname{mos} 47, \mathrm{XY},+\operatorname{mar} \operatorname{dn}[8] / 46, \mathrm{XY}[12])$. No major anomalies or dysmorphic features were observed at the age of 2 months. The psychomotor development of the boy was normal, and he reached all developmental milestones in due time. His phenotype showed no abnormality, and growth parameters were normal till the last examination at 4 years of age.

Molecular Cytogenetic Diagnostics of Marker Chromosomes
Case 2

The prenatal cytogenetic result was mos $46, \mathrm{X},+\operatorname{mar} \mathrm{dn}[9] /$ $45, \mathrm{X}[3] / 46, \mathrm{XX}[38]$. The $\mathrm{sSMC}^{\mathrm{T}}$ was confirmed to be a derivative of the X chromosome (Fig. 1B), and the mosaic ratio was refined using FISH to $26.6 \%$ of monosomic cells, $25.1 \%$ of cells with the $\mathrm{sSMC}^{\mathrm{T}}$, and $48.3 \%$ cells with a normal karyotype (in 627 cells examined). aCGH revealed the presence of $16.1 \mathrm{Mb}$ of the pericentromeric part of Xq $(\operatorname{mos} 46, X,+\operatorname{mar} \operatorname{dn}[9] / 45, X[3] / 46, X X[38]$. arr[GRCh37]Xq11.1q21.1(62057705_78218276) ×3 [0.2]) (Fig. 2A). Genes present on the sSMCs are shown for all patients in whom aCGH was performed in online suppl. Table 2.

Echocardiographic examination of the fetus in the 22nd week of gestation was normal. The parents accepted the risk of an unpredictable degree of Turner phenotype in the child and decided to continue the pregnancy. An apparently healthy girl was born in the 39th gestational week with AS of 9-10-10. The karyotype was confirmed postnatally, with a significantly higher proportion of cells with a normal karyotype ( $91.5 \%$ of 260 cells). Additional postnatal examinations revealed no major anomalies. Foramen ovale apertum was detected during a cardiologic examination in the infant period, and it has closed spontaneously before 3 years of age. The administration of growth hormone was initiated because of growth retardation at the age of 16 months. Till the age of 4 years, no remarkable facial dysmorphism was present and the

Cytogenet Genome Res 2018;154:187-195 
Fig. 2. aCGH results showing the extent of pericentromeric regions present in the sSMCs in cases 2 (A), 3 (B), and 4 (C). Duplication-safe (green), -critical (red), and -unknown (grey) regions according to Liehr [2014] are indicated for cases 3 and 4 (not applicable for $\mathrm{sSMC} \mathrm{C}^{\mathrm{T}}$ ). Our cases are shown as light blue bars, similar previously published cases mentioned in the text are shown as dark blue bars.

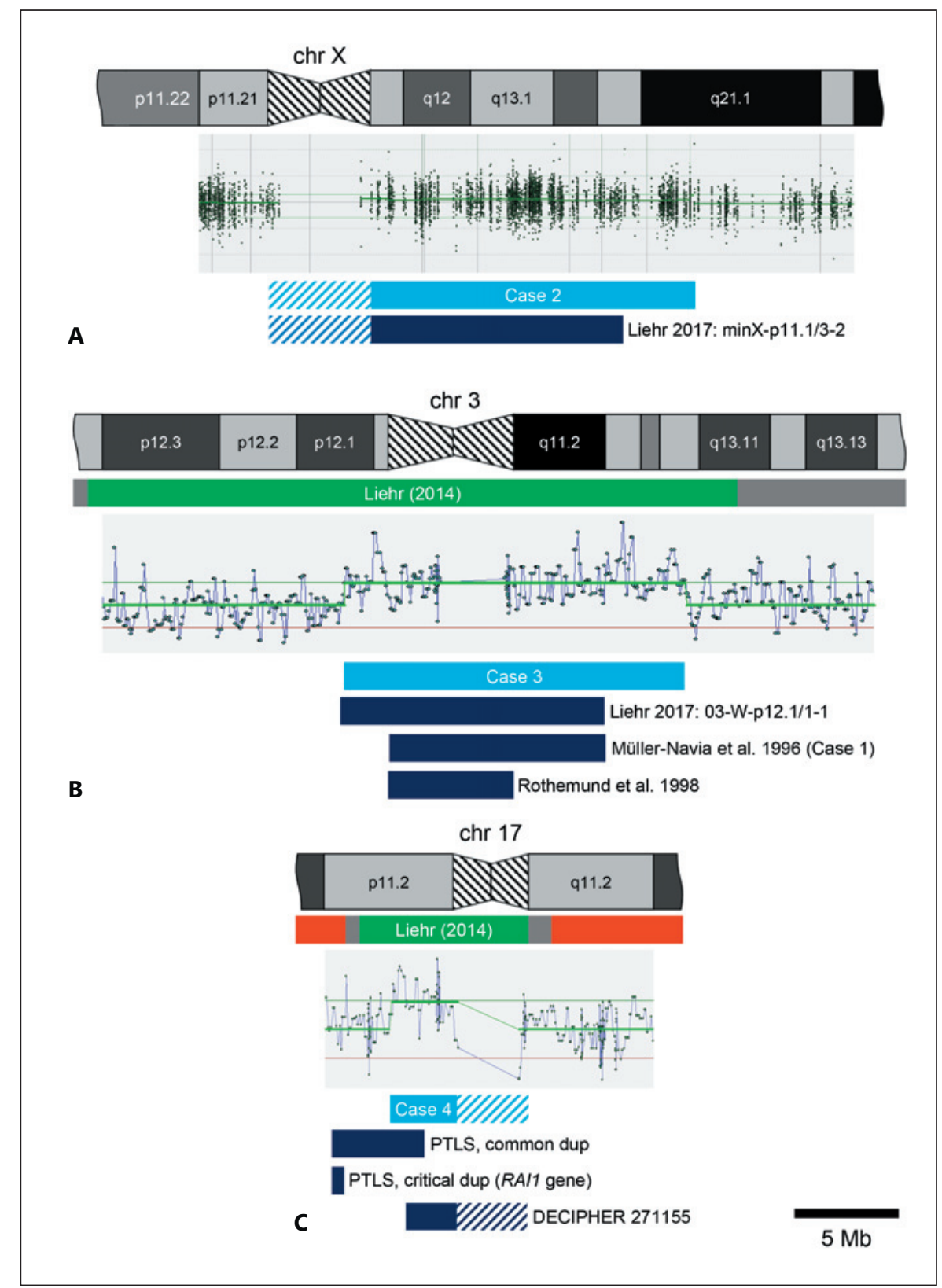

psychomotor development was adequate. The growth parameters normalized and the stature was proportional because of the growth hormone treatment.

Case 3

The prenatal karyotype was mos 47,XX,+mar dn[19]/46,XX[6]. An acrocentric origin of the sSMC was excluded, and it was found to be derived from chromosome 3 (Fig. 1C). FISH refined the mosaic ratio to $67 \%$ in 94 cells examined. aCGH demonstrated the presence of $4.9 \mathrm{Mb}$ of DNA from $3 \mathrm{p}$ and $9.2 \mathrm{Mb}$ from 3q $(47, X X,+$ mar dn[19/25].arr[GRCh37]3p12.1q12.3(85362707_ 102795128) $\times 3[0.7])$ (Fig. 2B).
The couple was consulted with a relatively favorable although still very uncertain prognosis, and they decided to continue the pregnancy, accepting the risk. A healthy girl was born in term with AS of 10-10-10. The prenatal finding was confirmed by postnatal karyotyping, with a lower degree of mosaicism (mos 47,XX,+der(3) $\mathrm{dn}[18] / 46, \mathrm{XX}[17])$. At the age of 1 year the development of the girl was appropriate, as well as her growth. No major anomalies were detected. During regular routine check-up at 3 years of age she was evaluated as a healthy child with adequate development without any peculiar features in appearance or behavior. She has required no special care and has exhibited no abnormality till the last examination at the age of 4 years. 
Fig. 3. Scheme of our algorithm for the determination of the origin of the sSMC. Horizontal arrows indicate a positive result of the examination and conclusion, vertical arrows indicate a negative result of the examination and proceeding to the next step. Blue lines indicate UPD testing: solid lines $=$ obligatory, dashed lines $=$ optional. a, alpha-satellite (centromeric) probe; wcp, whole chromosome painting probe. The colors of probe names indicate suggested labeling.

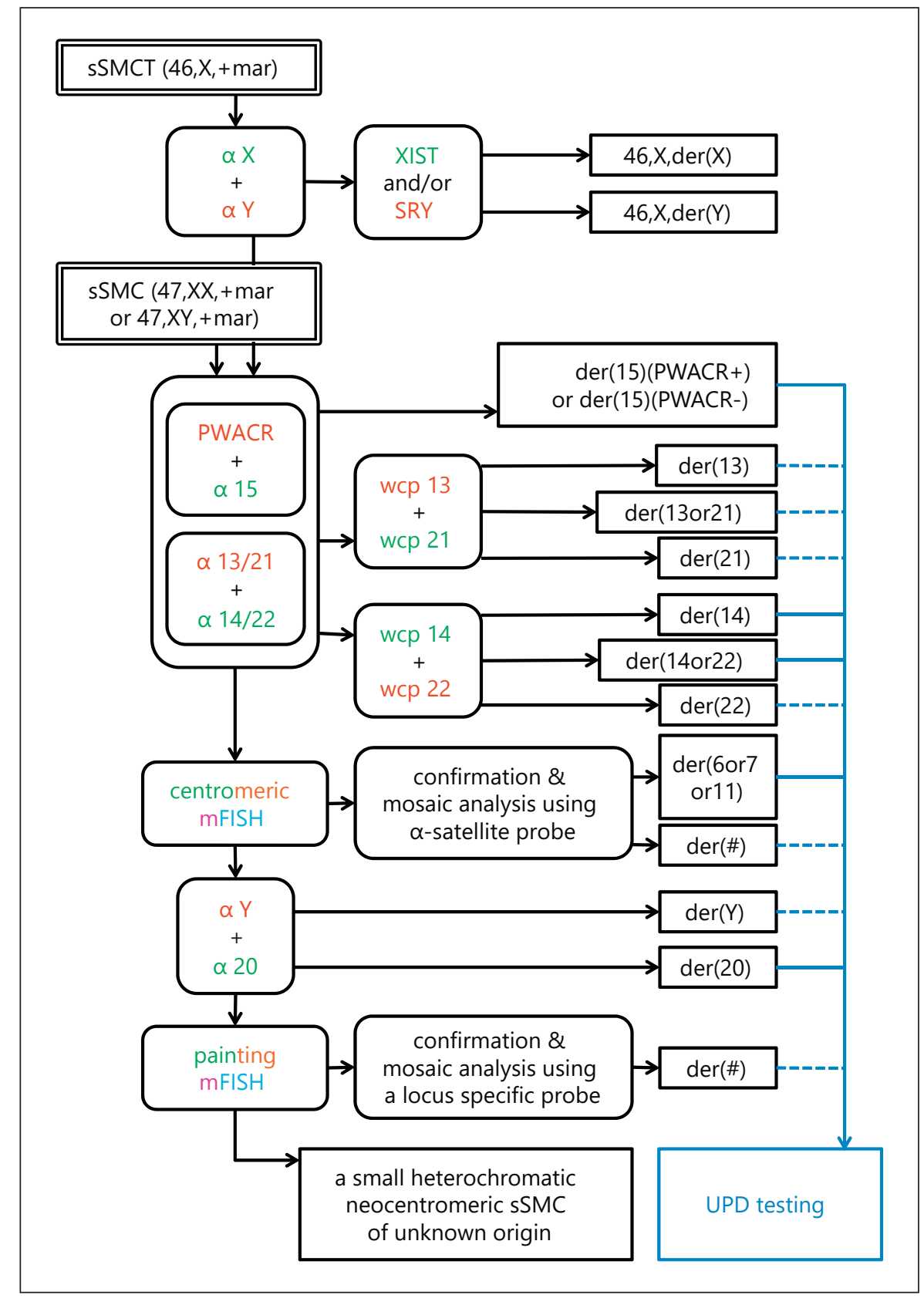

Case 4

The pregnancy was recognized by the mother in the 22nd week of gestation. Prenatal analysis revealed an abnormal karyotype $(\operatorname{mos} 47, \mathrm{XX},+\operatorname{mar} \operatorname{dn}[32] / 46, \mathrm{XX}[8])$. The origin of the sSMC was determined using cen-mFISH and confirmed using the alphacentromeric FISH probe. The finding was refined as mos 47,XX,+mar dn[32/40].ish der(17)(D17Z1+,D17S447+,D17S2027-, D17S1238-)[235/345]. The sSMC thus contained also a part of the common region of the Smith-Magenis syndrome and Potocki-Lupski (17p11.2 microduplication) syndrome (PTLS) (Fig. 1D).

The parents were informed about the uncertain prognosis associated with a $\operatorname{sMC}(17)$ and they decided to terminate the preg-

Molecular Cytogenetic Diagnostics of Marker Chromosomes nancy in the 23rd week of gestation. Autopsy of the fetus did not reveal any malformations. aCGH identified the sSMC as a $4.0 \mathrm{Mb}$ long segment of 17p (arr[GRCh37] 17p11.2p11.1(18184071_ 22226262) $\times 3[0.4])($ Fig. 2C).

\section{Materials and Methods}

sSMC cases 1-4 were identified during prenatal testing performed because of advanced maternal age in healthy Czech women aged $44,39,37$, and 38 years, respectively. The 4 couples had no remarkable family history of disease. No complications were re- 
ported during the 4 pregnancies, and the ultrasound examinations and biochemical screening yielded normal findings. Prenatal karyotyping was performed using standard protocols in cultivated amniocytes (cases 1-3) or fetal lymphocytes (case 4). Postnatal karyotyping was performed in peripheral lymphocytes. Karyotypes were evaluated according to ISCN [2016] as well as all results of FISH and aCGH.

sSMCs were characterized using FISH following the algorithm shown in Figure 3. sSMCs in cases 1, 3, and 4 were examined using centromeric probes for acrocentric chromosomes. This was followed by painting probes for chromosomes 13 and 21 in case 1 and by multicolor centromeric FISH (cen-mFISH; MetaSystems, Altlussheim, Germany; the probe is available upon request) followed by centromeric probes for chromosomes 3 and 17 (and 3 chromosome 17 locus-specific probes) in case 3 and 4, respectively. Case 2 was examined using probes for the sex chromosomes. FISH analyses employed commercially available probes (online suppl. Table 3) following the manufacturer's protocols. The Olympus BX51 microscope (Olympus, Tokyo, Japan) and Isis image analysis software (MetaSystems) were used for the FISH examinations.

Genomic DNA from cases 2-4 was analyzed using aCGH to specify the size of the sSMCs. CytoSure Chromosome X Array $4 \times 44 \mathrm{~K}$ (Oxford Gene Technology, Begbroke, UK), CytoChip ISCA $8 \times 60 \mathrm{~K}$ v2.0 (BlueGnome/Illumina, Cambridge, UK), and SurePrint G3 ISCA V2 Unrestricted $4 \times 180 \mathrm{~K}$ (Agilent Genomics, Santa Clara, CA, USA) arrays were used for cases 2-4, respectively. The genomic coordinates are based on human genome build hg19/ GRCh37.

Data on cases 1-4 were deposited in the SSMC database [Liehr, 2017] under accession numbers 13/21-O-q11/2-15, uminX-10, 03-O-p12.1/3-1, and 17-U-13, respectively.

\section{Results and Discussion}

Our case series describes the prenatal findings of 4 different types of de novo sSMCs of acrocentric, nonacrocentric, and gonosomal origin, which had a various size, gene content, and degree of mosaicism. The report illustrates the integration of the laboratory, clinical, and literature data to reach a prognosis and to allow proper counseling for the parents and their informed decision making.

The first mandatory step in the assessment of prognosis is to examine if the sSMC is de novo or if it was inherited from an unaffected or affected parent. De novo sSMCs are $2.5 \times$ more frequent than inherited sSMCs [Starke et al., 2003; Crolla et al., 2005]. If the sSMC is also present in a parent and if the level of mosaicism is comparable, the phenotype of the child is expected to be similar to that of the parent, although variable expressivity and a different degree of mosaicism in different tissues may alter the outcome. If the level of mosaicism differs substantially, the prognosis is less clear [Liehr et al., 2013]. As the second step, it is essential to specify the chromo- somal origin of the sSMC and to estimate its size and gene content. The prognosis for a de novo sSMC depends substantially on its chromosomal origin [Graf et al., 2006]. The degree of mosaicism must also be assessed, as 55$59 \%$ of sSMCs are present in a mosaic state [Starke et al., 2003; Crolla et al., 2005], and mosaicism may be even more frequent (70\%) among prenatal cases [Gruchy et al., 2008]. Finally, the findings must be compared with similar cases reported previously. The sSMC database [Liehr, 2017] is very helpful in finding analogical cases, but the original papers must be consulted for details. Other databases of copy number variation are also useful, e.g., DECIPHER, ECARUCA, ISCA, or DGV [de Leeuw et al., 2012], although they often contain insufficient clinical data, and contacting the contributing authors can also be more difficult compared to published papers. It is important to highlight that affected patients with sSMCs are more frequently examined and are more likely to be reported than unaffected carriers. Therefore, there is more information in the literature about sSMCs associated with a poor prognosis compared to those associated with a favorable outcome. Furthermore, the clinical assessment is frequently just performed in newborns or infants when many disorders may not be expressed yet. This is why reporting of every single case of a rare genetic abnormality is valuable and could help future clinicians and parents to reach proper prognosis assessments and reproductive decisions.

Our case 1 is an example of a carrier of an SSMC derived from an acrocentric chromosome. These are, apart from sSMCs(15)(PWACR+), generally associated with a favorable prognosis. Specifically, 112 carriers of centric sSMCs, the origin of which from chromosomes 13 or 21 could not be distinguished, were reported with sufficient clinical information, and 98 of them (87.5\%) were unaffected. The predominant symptoms in the remaining 14 individuals were dysmorphic features, in 6 cases with intellectual disability (ID). Notably, mainly cases with sSMCs with inverted duplication causing partial tetrasomy were present in the latter group [Liehr, 2017]. Only 2 cases with an sSMC of acrocentric origin without the inverted duplication structure had more severe abnormalities (heart and neural tube defects in a fetus, and partial seizures and moderate ID in a boy). However, these cases were examined only using centromeric probes, and it is not clear how much euchromatin their sSMCs contained [Hou et al., 1994; Brøndum-Nielsen and Mikkelsen, 1995]. These figures clearly illustrate the overall good prognosis for carriers of these sSMCs [Crolla et al., 1998], and our case 1 also showed no impairment. Because of
192

Cytogenet Genome Res 2018;154:187-195 DOI: $10.1159 / 000488790$
Tesner et al. 
this and of apparent lack of euchromatin on the sSMC, aCGH was not performed in this case.

Case 2 was a female with a derivative chromosome $\mathrm{X}$ supernumerary to the karyotype of Turner syndrome. The $45, \mathrm{X} / 46, \mathrm{X},+$ mar constitution is rare. No such case was detected among 4,269 pregnancies with ultrasound abnormalities. In patients with developmental delay and/or ID this karyotype was present in 9/15,053 cases $(1: 1,673$ or $0.060 \%)$ and in patients with infertility in $12 / 26,938$ cases $(1: 2,245$ or $0.045 \%)$ [Liehr et al., 2007]. Almost all these $\mathrm{sSMC}^{\mathrm{T}} \mathrm{s}$ originated from the chromosomes $\mathrm{Y}(72.6 \%)$ or $\mathrm{X}(27.0 \%)$ and were frequently associated with Turner syndrome phenotypes [Liehr et al., 2007]. It is impossible to determine the degree of mosaicism in various tissues of the fetus, but the relatively high proportion of amniocytes with a normal karyotype in case $2(48.3 \%)$ could be a favorable prognostic sign. FISH demonstrated the presence of the XIST locus on the $\mathrm{sSMC}^{\mathrm{T}}$, and thus its preferential inactivation is possible. The postnatal karyotype revealed a much higher proportion of cells with a normal karyotype (91.5\%). This was in accord with the normal development of case 2 and absence of abnormalities with the possible exception of growth retardation. The sSMC database contains a very similar case of a girl with short stature and $46 \%$ mosaic of $\min (\mathrm{X})(: \mathrm{p} 11.1 \rightarrow \mathrm{q} 13.3:)(\mathrm{XIST}+)$ [Liehr, 2017].

Case 3 carried an $\operatorname{SSMC(3).~So~far,~} 31$ cases with a centric $\operatorname{SSMC(3)}$ and sufficient clinical information have been described, 21 without any abnormality and 10 with an abnormal phenotype, mostly developmental delay [Liehr, 2017]. The material present on the SSMC(3) of case 3 originated from the region $3 \mathrm{p} 12.2 \mathrm{q} 13.11$, which is predicted not to be sensitive to a gain in copy number [Liehr, 2014]. All clinical findings indicated that the girl was unaffected. On the other hand, patients with similar sSMCs and abnormal phenotypes have also been reported: a fetus with dolichocephaly, oligohydramnios, and growth retardation spontaneously aborted in the 21 st week of gestation (case 03-W-p12.1/1-1) [Liehr, 2017], and a 1-year-old girl with a $70 \%$ mosaic and mild dysmorphic features, muscular hypotonia, and feeding difficulties, but with normal psychomotor development [Müller-Navia et al., 1996, case 1]. A 5-year-old girl has been described with mosaicism of one sSMC(3) in $36 \%$ of the cells and 2 sSMCs in $50 \%$ of the cells who exhibited developmental and growth delay and slight facial dysmorphism [Rothemund et al., 1998]. This could be caused by the second sSMC and partly by cerebellar astrocytoma at the age of 1 year. The parents of case 3 decided to continue the pregnancy, and no pathological symptoms could be observed in the child. She

Molecular Cytogenetic Diagnostics of Marker Chromosomes had neither seizures nor hypertrichosis as described in some patients with sSMC(3) [Liehr, 2017].

Case 4 carried a der(17). The sSMC database reports 33 cases of sSMC(17) with sufficient clinical information [Liehr, 2017]. Among them, 5 normal carriers have minute sSMCs spanning a small pericentromeric region (not exceeding 17p11.2q11.2) which is considered doseinsensitive [Liehr, 2014]. The other 28 individuals were affected (e.g., with developmental delay, facial dysmorphism, and hypotonia) [Liehr, 2017]. The pregnancy in case 4 was terminated, and no gross structural defects were found in the fetus. The sSMC covered the proximal two-thirds of the common PTLS region, but the RAI1 gene [Zhang et al., 2010] was not encompassed. PTLS is associated mainly with ID, autism, and hypotonia, which are not ascertainable by fetal autopsy. The aCGH result obtained from the autopsy of case 4 indicated that the sSMC originated from a region possibly not sensitive to a gain in copy number [Liehr, 2014]. On the other hand DECIPHER lists a case (271155) of a 30-year-old male with macrocephaly, developmental delay, and autism and a shorter 3-Mb long dup(17)(p11.2p11.1). The outcome in case 4 remains unknown because the pregnancy was terminated, and the correlation of her phenotype with other patients is impossible.

Our case series illustrates the utility of the diagnostic algorithm used, mainly in its speed, which is always needed in prenatal investigation. The algorithm is based on the frequency of sSMCs derived from different chromosomes. In cases with a prenatal karyotype $46, \mathrm{X}$, +mar carrying $\mathrm{sSMC}^{\mathrm{T}} \mathrm{s}$ (11.2\% of sSMC cases) [Liehr, 2017]), sex chromosomes should be analyzed first. This will result in the identification of $\operatorname{der}(\mathrm{X})$ or $\operatorname{der}(\mathrm{Y})$ in more than $99 \%$ of such cases [Liehr et al., 2007]. Only 3 cases of autosomal origin of $\mathrm{sSMC}^{\mathrm{T}}$ have been reported [Thangavelu et al., 1994; Gray et al., 2001; Sheth et al., 2009]. When the karyotype is $47, \mathrm{XX},+$ mar or $47, \mathrm{XY},+$ mar, the examination of acrocentric chromosomes should be performed first. We used 2 parallel hybridizations with mixes of centromeric probes for chromosomes 13/21 and 14/22, and probes for centromere 15 and PWACR. This step can characterize almost half of all sSMCs [Liehr, 2017]. sSMCs derived from chromosomes 13, 14, 21, and 22 can be further examined using whole chromosome painting probes. If the sSMC is of other than acrocentric origin, cen-mFISH for chromosomes 1-12, 15-19, and X should be used. The cen-mFISH result must be verified using the respective centromeric probe to exclude errors and to determine the degree of mosaicism. Overall, this algorithm can determine quickly (in 1-2 days) the origin of $95.8 \%$

Cytogenet Genome Res 2018;154:187-195 DOI: $10.1159 / 000488790$ 
sSMCs [Liehr, 2017]. Centromeric probes for the remaining chromosomes (20 and Y) and mFISH to identify neocentromeric sSMCs can be added if no result was reached.

Our algorithm simplifies and speeds up the procedure described by others, e.g., that proposed by Liehr et al. [2009]. We replaced the NOR staining (which is followed by centromeric FISH for acrocentrics in the procedure by Liehr et al. [2009] anyway) by a fast direct FISH examination of acrocentric chromosomes and skipped the potentially problematic size comparison to chromosome 20 (as the origin of all marker chromosomes is identified in the first or second step). Many hours (or days in some cases) are saved by the replacement of examinations using individual centromeric or painting probes by multicolor FISH. Also compared to the most recent sSMC identifying algorithm by Jang et al. [2016], our algorithm investigates all acrocentric chromosomes at once, and it also enables detection of the less frequent sSMCs using cen$\mathrm{mFISH}$. This accelerates the diagnostic procedure and reduces the uncertainty, which is essential in prenatal diagnostics. Karyotyping of the parents is an indispensable part of all algorithms as well as the preferential testing of sex chromosomes in $\mathrm{SSMC}^{\mathrm{T}}$ cases.

A parallel examination using aCGH should always be engaged to expand the knowledge about the sSMC but also to exclude other submicroscopic copy number variants. FISH results should be available in the majority of cases for guiding the aCGH evaluation. In cases 3 and 4 , aCGH could determine the origin and size of the sSMCs and could be used as a stand-alone approach. When mosaicism is low, as in case 2, only the knowledge of the origin and mosaic ratio obtained using FISH can guide the interpretation of the aCGH result. Case 1 may represent an example of an sSMC formed exclusively by heterochromatin where aCGH is of limited use. Generally, aCGH is useful in the specification of genes present on the sSMC, which may have prognostic consequences if any of them is dosage sensitive. Single nucleotide polymorphism arrays (SNPa) could be used for uniparental disomy (UPD) testing and assessment of the parental origin of the amplified material in one step and thus shed light onto the mechanism of formation of the sSMC as shown by Hochstenbach et al. [2013]. If SNPa is not used, UPD testing using other methods should be performed at least for sSMCs derived from chromosomes with known imprinted loci, i.e., derivatives of chromosomes $6,7,11,14,15$, and 20 [Mackay and Temple, 2017], which are also among those most frequently reported in association with UPD (chromosomes 6, 7, 14, 15, 16, and 20) [Liehr et al., 2011].
Our case series shows 4 cases of prenatally identified sSMCs representing a variable prognosis for their carriers, ranging from a very low risk associated with tiny sSMCs derived from acrocentrics over a possibly limited contribution of a sSMC ${ }^{\mathrm{T}}$ derived from the $\mathrm{X}$ chromosome on the background of gonosomal aneuploidy to a generally rather high risk of sSMCs derived from nonacrocentrics where the chromosome of origin, extent, gene content, and the degree of mosaicism of the sSMC play key roles. The availability of clinical and laboratory data from previously described carriers of similar sSMCs is very valuable, and reporting of every single case is helpful in the assessment of prognosis in future cases and for reaching proper informed decisions. The growing amount of information collected will continue to reduce the dilemmas inherently associated with these challenging diagnostic and counseling situations.

\section{Acknowledgments}

The authors wish to thank all families for participating in this study. This work was supported by grants No. 264811 from the Grant Agency of Charles University and TA01010931 from the Technology Agency of the Czech Republic.

\section{Statement of Ethics}

The research was ethically conducted in accordance with the World Medical Association Declaration of Helsinki. All subjects (or their parents or guardians) have given their informed written consent.

\section{Disclosure Statement}

The authors declare no conflicts of interest.

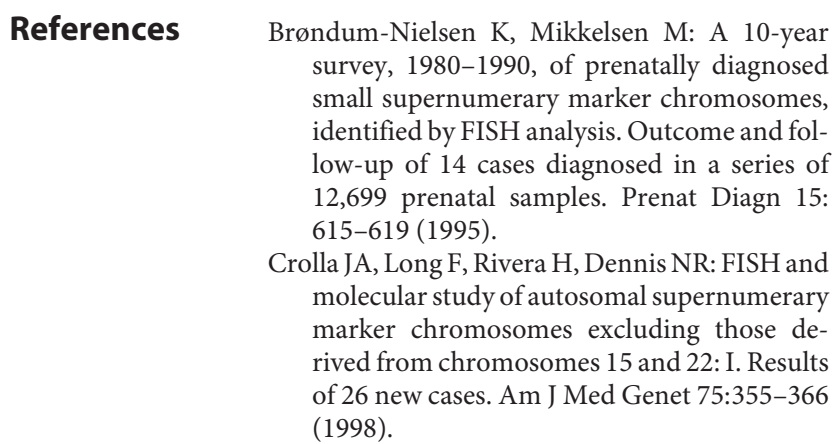

Tesner et al. 
Crolla JA, Youings SA, Ennis S, Jacobs PA: Supernumerary marker chromosomes in man: parental origin, mosaicism and maternal age revisited. Eur J Hum Genet 13:154-160 (2005).

$\checkmark$ de Leeuw N, Dijkhuizen T, Hehir-Kwa JY, Carter NP, Feuk L, et al: Diagnostic interpretation of array data using public databases and internet sources. Hum Mutat 33:930-940 (2012).

-Graf MD, Christ L, Mascarello JT, Mowrey P, Pettenati M, et al: Redefining the risks of prenatally ascertained supernumerary marker chromosomes: a collaborative study. J Med Genet 43:660-664 (2006).

Gray BA, Bent-Williams A, Wolff DJ, Zori RT: A non-sex chromosome marker in a patient with an atypical Ullrich-Turner phenotype and mosaicism of 46,X,mar/46,XX. Clin Genet 60:73-76 (2001).

-Gruchy N, Lebrun M, Herlicoviez M, Alliet J, Gourdier D, et al: Supernumerary marker chromosomes management in prenatal diagnosis. Am J Med Genet A 146A:2770-2776 (2008).

-Hochstenbach R, van Gijn ME, Krijtenburg PJ, Raemakers R, van 't Slot R, et al: Gain of FAM123B and ARHGEF9 in an obese man with intellectual disability, congenital heart defects and multiple supernumerary ring chromosomes. Mol Syndromol 3:274-283 (2013).

Hochstenbach R, Poot M, Liehr T: Mechanisms of origin and clinical effects of multiple small supernumerary marker chromosomes, each derived from a different chromosome. OBM Genetics 1:002; doi:10.21926/obm.genet. 1701002 (2017).

- Hou JW, Liu CH, Wang TR: Molecular cytogenetic studies of children with marker chromosomes. J Formos Med Assoc 93:205-209 (1994).

ISCN 2016: An International System for Human Cytogenetic Nomenclature, McGowan-Jordan J, Simons A, Schmid M (eds). Cytogenet Genome Res 149:1-140 (2016).

-Jang W, Chae H, Kim J, Son JO, Kim SC, et al: Identification of small marker chromosomes using microarray comparative genomic hy- bridization and multicolor fluorescent in situ hybridization. Mol Cytogenet 9:61 (2016).

Kleefstra T, de Leeuw N, Wolf R, Nillesen WM, Schobers G, et al: Phenotypic spectrum of 20 novel patients with molecularly defined supernumerary marker chromosomes 15 and a review of the literature. Am J Med Genet A 152A:2221-2229 (2010).

Kumar C, Kleyman SM, Samonte RV, Verma RS: Marker chromosomes in fetal loss. Hum Reprod 12:1321-1324 (1997).

Liehr T: Benign and Pathological Chromosomal Imbalances: Microscopic and Submicroscopic copy Number Variations (CNVs) in Genetics and Counselling. (Elsevier, Amsterdam 2014).

Liehr T: Small supernumerary marker chromosomes. http://ssmc-tl.com/sSMC.html [accessed 06/06/2017] (2017).

Liehr T, Weise A: Frequency of small supernumerary marker chromosomes in prenatal, newborn, developmentally retarded and infertility diagnostics. Int J Mol Med 19:719731 (2007).

Liehr T, Mrasek K, Hinreiner S, Reich D, Ewers E, et al: Small supernumerary marker chromosomes (sSMC) in patients with a 45,X/ $46, \mathrm{X},+$ mar karyotype -17 new cases and a review of the literature. Sex Dev 1:353-362 (2007).

Liehr T, Ewers E, Kosyakova N, Klaschka V, Rietz F, et al: Handling small supernumerary marker chromosomes in prenatal diagnostics. Expert Rev Mol Diagn 9:317-324 (2009).

Liehr T, Ewers E, Hamid AB, Kosyakova N, Voigt $\mathrm{M}$, et al: Small supernumerary marker chromosomes and uniparental disomy have a story to tell. J Histochem Cytochem 59:842-848 (2011).

Liehr T, Klein E, Mrasek K, Kosyakova N, Guilherme RS, et al: Clinical impact of somatic mosaicism in cases with small supernumerary marker chromosomes. Cytogenet Genome Res 139:158-163 (2013).

Mackay DJG, Temple IK: Human imprinting disorders: principles, practice, problems and progress. Eur J Med Genet 60:618-626 (2017).
Marle N, Martinet D, Aboura A, Joly-Helas G, Andrieux J, et al: Molecular characterization of 39 de novo sSMC: contribution to prognosis and genetic counselling, a prospective study. Clin Genet 85:233-244 (2014).

Müller-Navia J, Nebel A, Oehler D, Theile U, Zabel B, Schleiermacher E: Microdissection and DOP-PCR-based reverse chromosome painting as a fast and reliable strategy in the analysis of various structural chromosome abnormalities. Prenat Diagn 16:915-922 (1996).

Nietzel A, Rocchi M, Starke H, Heller A, Fiedler W, et al: A new multicolor-FISH approach for the characterization of marker chromosomes: centromere-specific multicolor-FISH (cenMFISH). Hum Genet 108:199-204 (2001).

Rothemund H, Greenberg CR, Dawson AJ: Mosaic supernumerary marker chromosome identified as a der(3) by FISH. Clin Genet 54: 526-527 (1998).

Sheth F, Ewers E, Kosyakova N, Weise A, Sheth J, et al: A small supernumerary marker chromosome present in a Turner syndrome patient not derived from X- or Y-chromosome: a case report. Mol Cytogenet 12:22 (2009).

Starke H, Nietzel A, Weise A, Heller A, Mrasek K, et al: Small supernumerary marker chromosomes (sSMCs): genotype-phenotype correlation and classification. Hum Genet 114:51-67 (2003).

Thangavelu M, Pergament E, Espinosa R 3rd, Bohlander SK: Characterization of marker chromosomes by microdissection and fluorescence in situ hybridization. Prenat Diagn 14:583-588 (1994).

Warburton D: De novo balanced chromosome rearrangements and extra marker chromosomes identified at prenatal diagnosis: clinical significance and distribution of breakpoints. Am J Hum Genet 49:995-1013 (1991).

Zhang F, Potocki L, Sampson JB, Liu P, SanchezValle A, et al: Identification of uncommon recurrent Potocki-Lupski syndrome-associated duplications and the distribution of rearrangement types and mechanisms in PTLS. Am J Hum Genet 86:462-470 (2010).
Molecular Cytogenetic Diagnostics of Marker Chromosomes
Cytogenet Genome Res 2018;154:187-195 DOI: $10.1159 / 000488790$ 